\title{
Systematic survey on evolution of cloud architectures
}

\section{S. Bharath Bhushan* and Pradeep Reddy}

School of Information Technology and Engineering,

VIT University, Vellore, India

Email: bharath.bhushan4@gmail.com

Email: pradeep1417@gmail.com

*Corresponding author

\section{Dhenesh V. Subramanian}

School of Computing Information and Mathematical Sciences, University of the South Pacific, Suva, Fiji

Email: vsdhenesh@gmail.com

\section{X.Z. Gao}

Department of Electrical Engineering and Automation, Aalto University School of Electrical Engineering, Aalto, Finland

Email: xiao.z.gao@gmail.com

\begin{abstract}
Cloud architectures are becoming an active area of research. The quality and durability of a software system are defined by its architecture. The architecture approaches that are used to build cloud-based systems are not available in a blended fashion to achieve an effective universal architecture solution. The paper aims to contribute to the systematic literature review (SLR) to assist researchers who are striving to contribute in this area. The main objective of this review is to systematically identify and analyse the recently published research topics related to software architecture for cloud with regard to research activity, used tools and techniques, proposed approaches, domains. The applied method is SLR based on four selected electronic databases proposed by (Kitchenham and Charters, 2007). Out of 400 classified publications, we regard 121 as relevant for our research domain. We outline taxonomy of their topics and domains, provide lists of used methods and proposed approaches. At present, there is little research coverage on software architectures for cloud, while other disciplines have become more active. The future work is to develop a secure architecture to achieve quality of service and service level agreements.
\end{abstract}

Keywords: cloud computing; software architectures; quality of service; service level agreement; resource and service management; security.

Reference to this paper should be made as follows: Bhushan, S.B., Reddy, P., Subramanian, D.V. and Gao, X.Z. (xxxx) 'Systematic survey on evolution of cloud architectures', Int. J. Autonomous and Adaptive Communications Systems, Vol. X, No. Y, pp.xxx-xxx. 
Biographical notes: S. Bharath Bhushan received his Bachelors in Information Technology from the JNTUA, Andhra Pradesh, India in 2011 and his Master in Computer Networks and Information Security from the same university in 2013. From 2012 to 2013, he was an Intern with Integra Micro Software Services Pvt. Ltd., Bangalore, India. From 2013 to 2014, he was an Assistant Professor with Sree Vidyanikethan Engineering College., Tirupathi, India. $\mathrm{He}$ is currently a Research Scholar with VIT University, Vellore, India. His research interests include cloud computing distributed computing and networks.

Pradeep Reddy is currently working with the School of Information Technology and Engineering, VIT University, Vellore, India. He has a total of eight years of experience in both teaching and research. He received his BTech in Computer Science and Engineering from PBR VITS, JNTU in 2004, Andhra Pradesh, India and MTech in Computer Science and Engineering from VIT University, Vellore, India. $\mathrm{He}$ did his $\mathrm{PhD}$ in Computer Science and Engineering from VIT University, Vellore in 2014. His research interests include mobile and wireless systems and cloud computing.

Dhenesh V. Subramanian received his Doctoral in Information Technology from the University of South Australia, Adelaide. He completed his Masters in Computer Applications and Bachelor's in Physics from Bharathiar University, Coimbatore, India. He has eight years of teaching and four years of research experience in various institutions in India and Australia. He is currently working as an Associate Professor with VIT University, India. His research interest includes software engineering and cloud computing.

X.Z. Gao received his DSc (Technology) degree from the Helsinki University of Technology (now Aalto University), Finland in 1999. He is currently working as a Docent in the Department of Electrical Engineering and Automation, Aalto University School of Electrical Engineering, Finland. His research interests are nature-inspired computing methods with applications in optimisation, data mining, industrial electronics and control systems.

\section{Motivation and background}

Cloud architectures are becoming an active area of research. As we know the architecture plays a crucial role in the design and development of software systems. A good architecture can ensure that a system will satisfy key requirements in such topics as performance, reliability, scalability and interoperability. In spite of this, the on-demand, scalable and rapid provisioning nature of cloud poses serious obstacles to any architecture. So the task is to design and develop an effective security architecture that will support cloud environment. As of now there is little contribution to systematic literature review for mapping software architectures and cloud environment.

This literature review aids researcher who is ambitious to contribute in this area, without investing time in doing a detailed literature survey.

\subsection{Introduction to cloud computing}

Cloud computing is an emerging new computing paradigm for delivering IT services, cloud computing became an attractive option for cloud service providers and consumers. Cloud is a pool of shared resources which can be served rapidly with minimal 
management (Blank, 2011). The main cloud services are software; platform and infrastructure are provided as services. The deployment models are private cloud, public cloud, hybrid cloud and community cloud. The key feature of this computing is the ability to deliver services as pay per use basis. Service level agreements are used for specification of QoS (Duan et al., 2012) requirements between cloud service providers and cloud service users, which helps to meet quality of service. However, software as a service refers to applications that are delivered to customers over the network on the basis of pay-as-you-go. The idea behind platform as a service is to provide a software development platform as a service, including deployment, execution and testing. The infrastructure as a service provides computational infrastructure as a service which are processed, desktop, storage and a lot more. A cloud environment that is shared among different clients is called public cloud, the infrastructure which is owned and maintained by an organisation will provide as services on the basis of billing. Some organisations build or rent to have an own cloud infrastructure because of security reasons. This is called private cloud, operated exclusively for an organisation. Hybrid cloud is a combination of private and public cloud, where part of an application runs on a public cloud and other part runs on a private cloud. If two or more organisations have similar interests they build or rent dedicated environment, then it is community cloud (Buyya et al., 2009; Ali et al., 2015). To conclude, no other technology has shown greater impact as cloud computing on IT industry, research and academics.

\subsection{Software and cloud architectures}

The software architecture of a computing system is a representation of the system that helps in understanding of how the system will work. A good architecture can help and ensure that a system will satisfy key requirements in such topics as performance, reliability, portability, scalability, and interoperability. The software architecture of a system is the set of structures needed to reason about the system, which comprise software elements, relations among them, and properties (Bass and Kazman, 2012). So architectures play a crucial role in the design and development of software systems.

Cloud architecture aids in the design and development of cloud application which must be scalable, on-demand, automated and ubiquitous services. The application which runs on cloud infrastructure should utilise the resources when it requires and release them after a job is finished and the resources can be elastically provisioned based on application demand. The cloud architectures must ensure the provisioning of services, security, load balancing, scheduling, service level agreement, quality of service and lot more. However, rapid provisioning and flexibility that cloud computing offers to pose serious obstacles to any cloud architecture.

The NIST cloud reference architecture is a high level architecture which is not specific to any cloud vendor that helps in discussing the requirements, structures and operations of cloud (Liu et al., 2011). It defines a set of actors, activities, methods and standards in order to develop cloud architecture. The actors are cloud consumer, cloud provider, cloud broker, cloud auditor, and cloud carrier which helps to gather functional and non-functional requirements from stakeholders to develop cloud enabled application satisfying service level agreements and quality of service. The IBM cloud reference architecture (Stifani et al., 2012) is also widely accepted architecture which presents three main roles which are cloud service provider, cloud service consumer and cloud service creator. 


\subsection{Research questions}

The main intention of this paper was to find and interpret the published literature related to software architecture and cloud environment. This is further detailed in the following research questions:

RQ1 How much activity was carried out in the last four years?

RQ2 What research topics are being addressed?

RQ3 What are the different tools, standards and technologies that were used?

RQ4 What are the different application domains/case studies implemented?

RQ5 What are the different cloud setups that have been adopted?

\subsection{Related work}

There are systematic literature reviews on different topics in cloud computing. And Chauhan (2014) conducted a systematic mapping of software architectures for cloud environment. They did a systematic literature review of journals and conferences from January 2008 to July 2011.

Since then, the number of publications in the domain has increased and we focused on a systematic literature review from January 2011 to August 2014 from all repositories with different search strings.

\section{Search strategy}

We performed our search on scientific electronic databases which includes high impact factor conferences, journals and articles. The search process follows the guidelines suggested by (Kitchenham and Charters, 2007). Refer to Table 1 for a list of selected electronic databases.

Table 1 Selected electronic databases

\begin{tabular}{lc}
\hline Electronic database & URL \\
\hline IEEE & $\mathrm{http}: / /$ ieeexplore.ieee.org \\
ACM & $\mathrm{http}: / / \mathrm{dl}$.acm.org \\
Science direct & $\mathrm{http} / / / \mathrm{www}$. sciencedirect.com \\
Springer & $\mathrm{http} / / / \mathrm{www}$. springerlink.com \\
\hline
\end{tabular}

\subsection{Search string}

Search string helps to capture all results related to cloud architectures and software architectures. The reasons for searching with cloud services and architecture as keywords is to ensure all relevant papers are included. The search string used on all databases is:

(Cloud architecture OR cloud framework OR cloud model OR cloud computing) AND (Software architecture) AND (Software as a service OR platform as a service OR infrastructure as a service) 


\subsection{Inclusion criteria and exclusion criteria}

In order to include relevant publications in our review, we defined selection criteria and based on that we performed inclusion and exclusion of published literature. We selected papers published in peer review conferences, journals from $1 / 1 / 2011$ to $18 / 8 / 2014$. We selected papers that are relevant to our research questions. We excluded papers that are not related to software architecture and cloud. Table 2 shows our inclusion and exclusion criteria.

Table 2 Inclusion and exclusion criteria

\begin{tabular}{lc}
\hline Inclusion criteria & Exclusion criteria \\
\hline $\begin{array}{l}\text { Publications from } 1 / 1 / 2011 \text { on software } \\
\text { architectures related to cloud. }\end{array}$ & Publications before 2011 and after 18/8/2014 \\
Papers published in journals and conferences & Articles, book reviews and editorials \\
Published in peer reviewed studies & Published in non-peer reviewed studies \\
$\begin{array}{l}\text { Publications that are related to research } \\
\text { questions }\end{array}$ & Architectures from general and other computer \\
\hline
\end{tabular}

\subsection{Roles and responsibilities}

- $\quad$ Bharath Bhushan (VIT, research scholar): result classification and detailed analysis for various journals and papers from IEEE, ACM, Science direct, and SpringerLink

- Dhenesh V Subramanian (VIT, expert reviewer): assessment of classification and detailed analysis

- $\quad$ Pradeep Reddy and Gao, expert reviewers: assessment of search result classification and detailed analysis.

\subsection{Conference and journal selection process}

The process was conducted as follows:

1 the researchers perform the search on each database and save the references in bibliography files

2 the scholar reads all titles and abstracts and checks the inclusion and exclusion criteria for each entry

3 the scholar classifies the conferences and journals according to type, topic, and domain

4 the expert reviewer reassesses the classification and inclusion/exclusion of search results.

\subsection{Data analysis}

The data is analysed to show:

1 the databases and number of query results 
2 the publications are listed as per databases with respect to authorship, reference, date, publication type, type of content, topic of content and domain

3 the number of relevant publications per year with respect to venues

4 the graph that will show publication of journals and conferences, which are generated from the final results

5 a detailed selection process performed on selected databases.

\section{Results}

The distribution of results for each database related to search criteria is listed in Table 3.

Table 3 Number of results per database

\begin{tabular}{lcc}
\hline Database & Search date & Results (\%) \\
\hline IEEE & $12 / 8 / 2014$ & 37.19 \\
ACM & $12 / 8 / 2014$ & 23.96 \\
Science direct & $14 / 8 / 2014$ & 27.27 \\
Springer & $14 / 8 / 2014$ & 11.57 \\
\hline
\end{tabular}

All results were ordered 'by relevance' as shown by the databases. From these results, we considered the first 100 results of each database in our first repetition of the study. In total we reviewed 400 publications.

The following acronyms are used to categorise the results in Tables 4-7.

- Publication: The included publications classified as journals, conference paper.

- Type: What kind of information was presented in the publication, e.g., method, model, review, tool, case study.

- Topic: The exact intention and purpose of the publication.

- Area: We classified publications into five areas, namely: resource management, service management, quality of service, security and cloud application. This will also ensure the publications are relevant to include in the review.

RQ1 How much activity was carried out in last four years?

We plotted a number of relevant publications per databases in Figure 2, per publication type in Figure 3 and per year in Figure 4. In the last four years, there was a noteworthy increase in number of publications compared with 2008 to 2011 that shows the significance of the review on cloud architectures. The first paper on cloud architectures was published in 2008 addressing architecture of cloud for applications. Kitchenham and Charters (2007) did a literature review on cloud architectures from 2008 to 2011, where a significant increase in number of publications that focused mainly on quality, multi-tenancy, frameworks, security and application domains. The papers mainly focused on horizontal research rather than a vertical approach. Figure 4 shows numbers of papers published from 2011 to 2014. 
However, there are only 18 papers in 2014. We performed searches in August 2014 and all the papers had not been available by that time it might be the reason for less number of papers in 2014.

The reviewed papers will help in building up a body of knowledge in cloud architectures.

Figure 1 Selection process

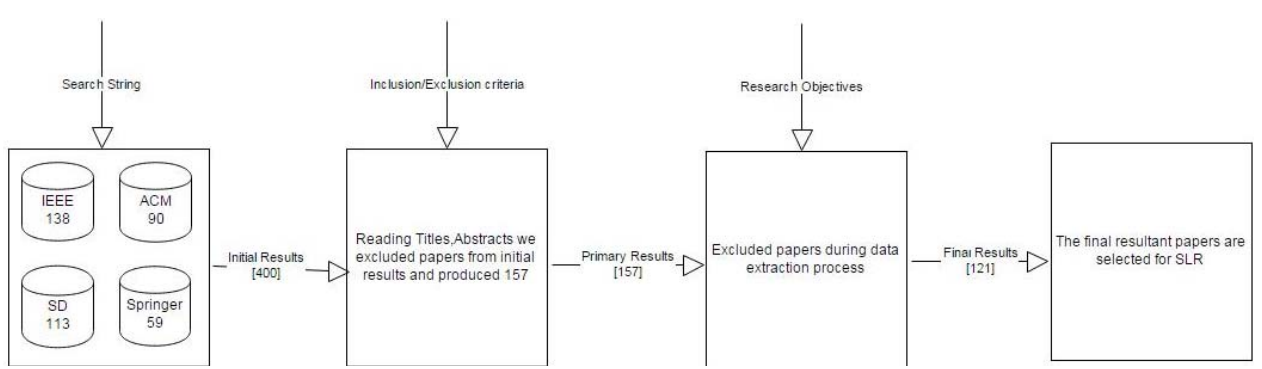

Figure 2 Included results per database

Total

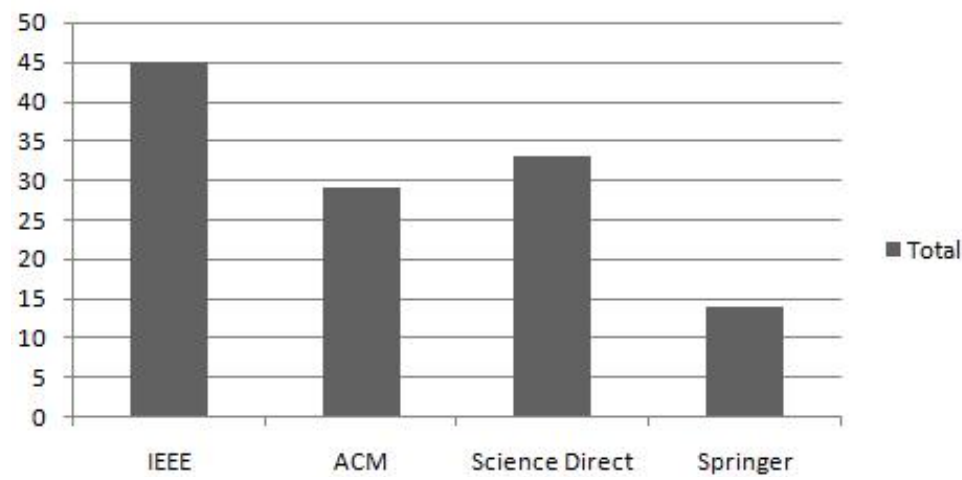

Figure 3 Included results per publication type (see online version for colours)

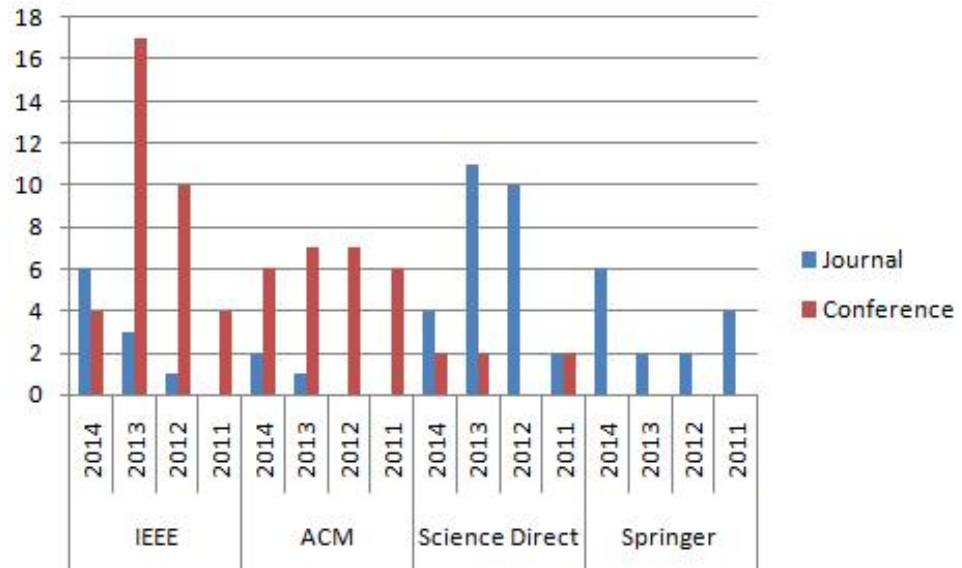


Figure 4 Included results per year (see online version for colours)

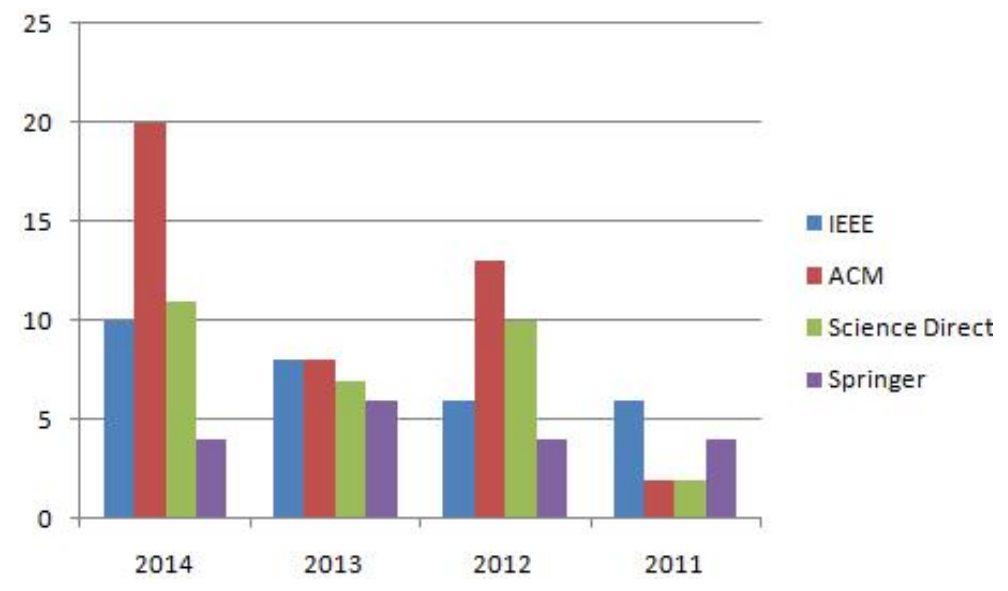

RQ2 What research topics are being addressed?

To know the research topics that are focused in cloud architectures, we have generated a weighted topic and taxonomy in Figure 5, which are derived from titles, keywords and topics are shown in Tables 4-7. The research topics are broadly classified into, resource management, service management, quality of service (QoS), security and cloud application domain.

The research topics that are considered under resource management deals with management of infrastructure like servers, storage, computing power, network, topics that deal with management of services that are delivered by the cloud service provider like cloud applications, infrastructure, platform and anything as a service is under resource management, topics that deal performance, reliability, availability, fault tolerance, incident response, SLA is under quality of service, topics that deal with cloud network, data security, trust management, secure architectures, secure services and a lot more are under security management, the papers that deal with cloud applications are under the application domain (Xu et al., 2014).

RQ3 What are the different tools, standards and technologies that were used?

There is a wide variety of tools and technologies being used in studies listed in Table 7. We classified them according to research topics where they were explicitly used. A possible reason for it can be, they tend to provide generic solutions. Eucalyptus, open nebula, nimbus and open stack tools are chosen by most of the researchers for cloud setup. Aneka, Greencloud and Cloudsim tools are used for building applications and managing resources in a cloud environment. Many of the researchers have chosen Java because of their platform independent nature and the virtualisation technologies they used like Xen, VMware and KVM. They have used Microsoft azure and Google app engine as a platform for their experiments. And few use Nagios for network, infrastructure monitoring and IBM Tivoli for network management (Vasilakos, 2008). Most of the studies adopted standards for communication and security. 
Table 4 Included results from IEEE Xplore

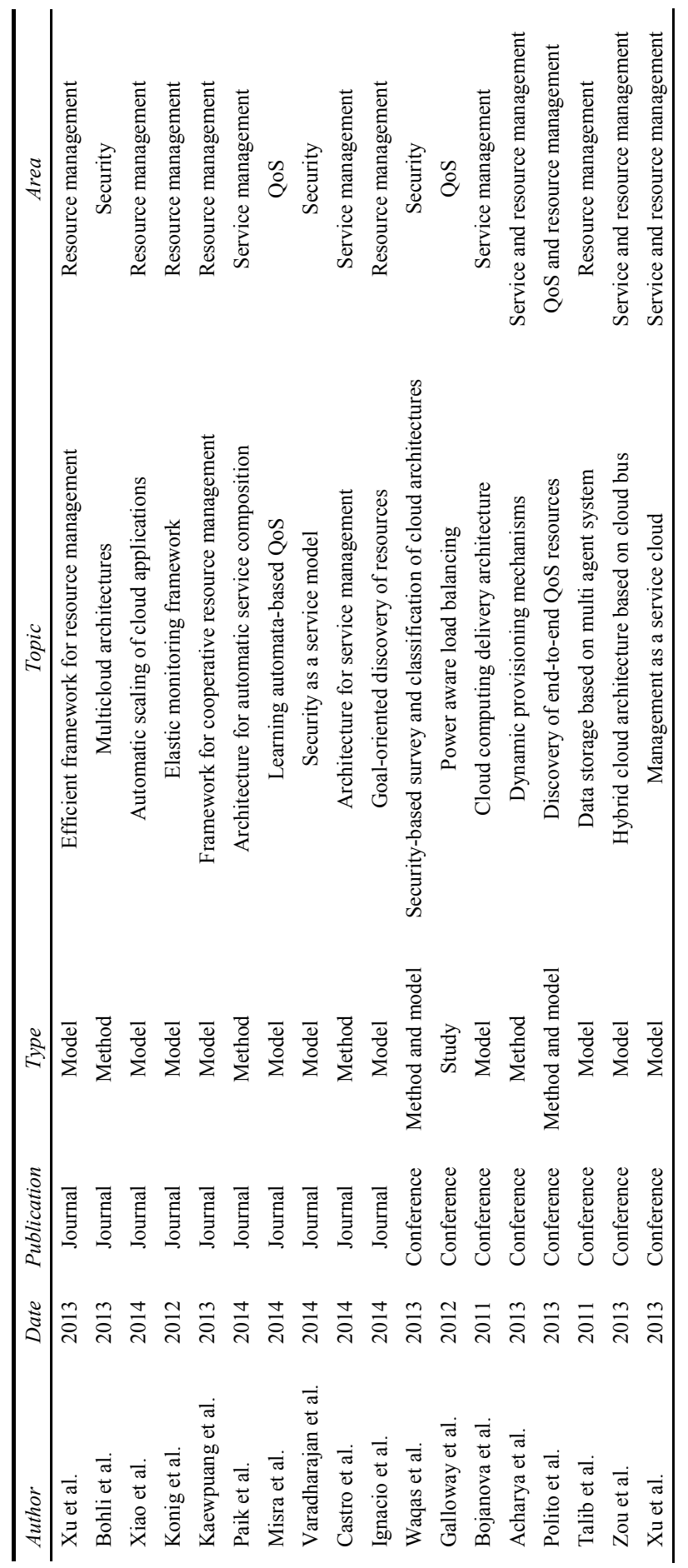


Table 4 Included results from IEEE Xplore (continued)

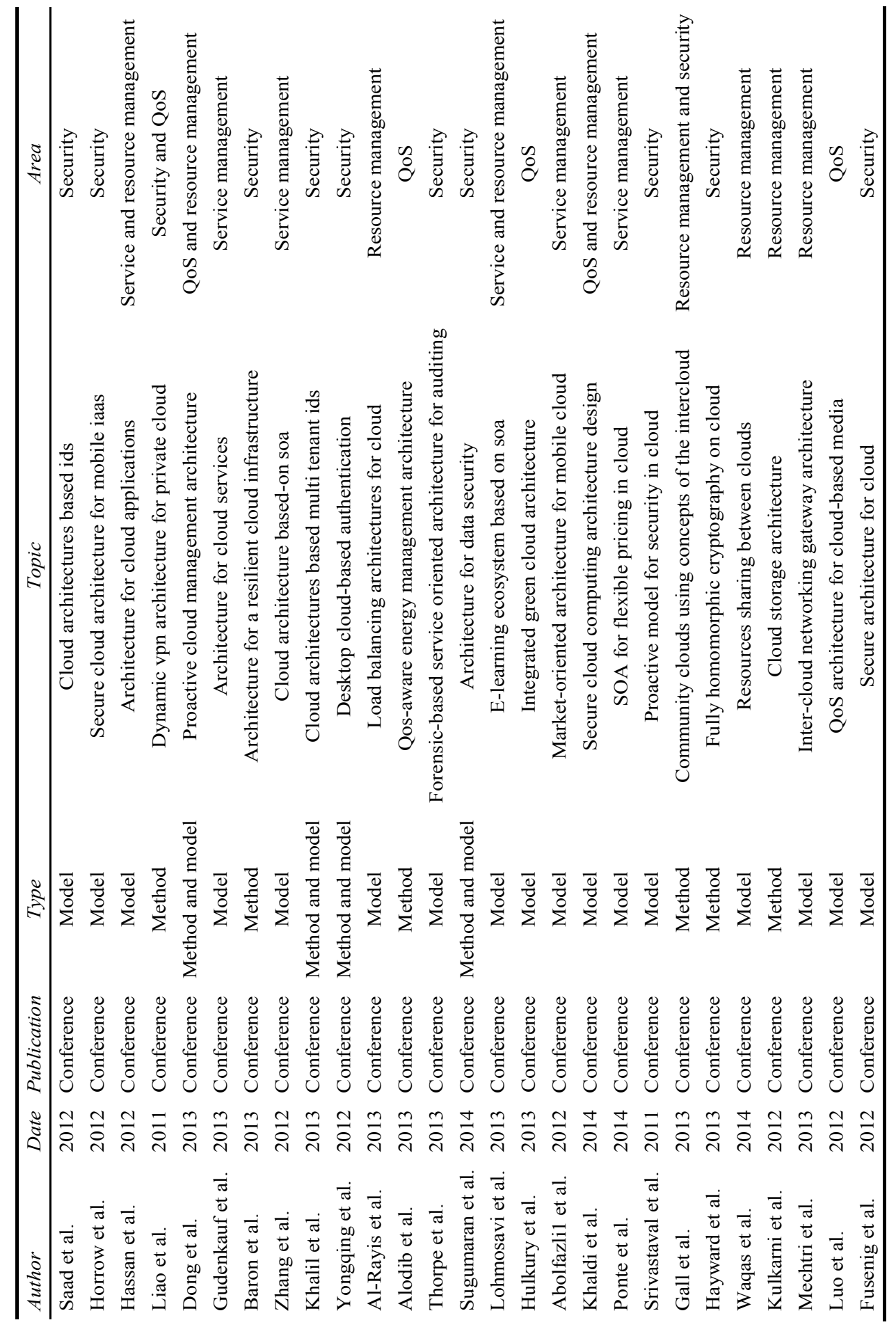


Table 5 Included results from ACM

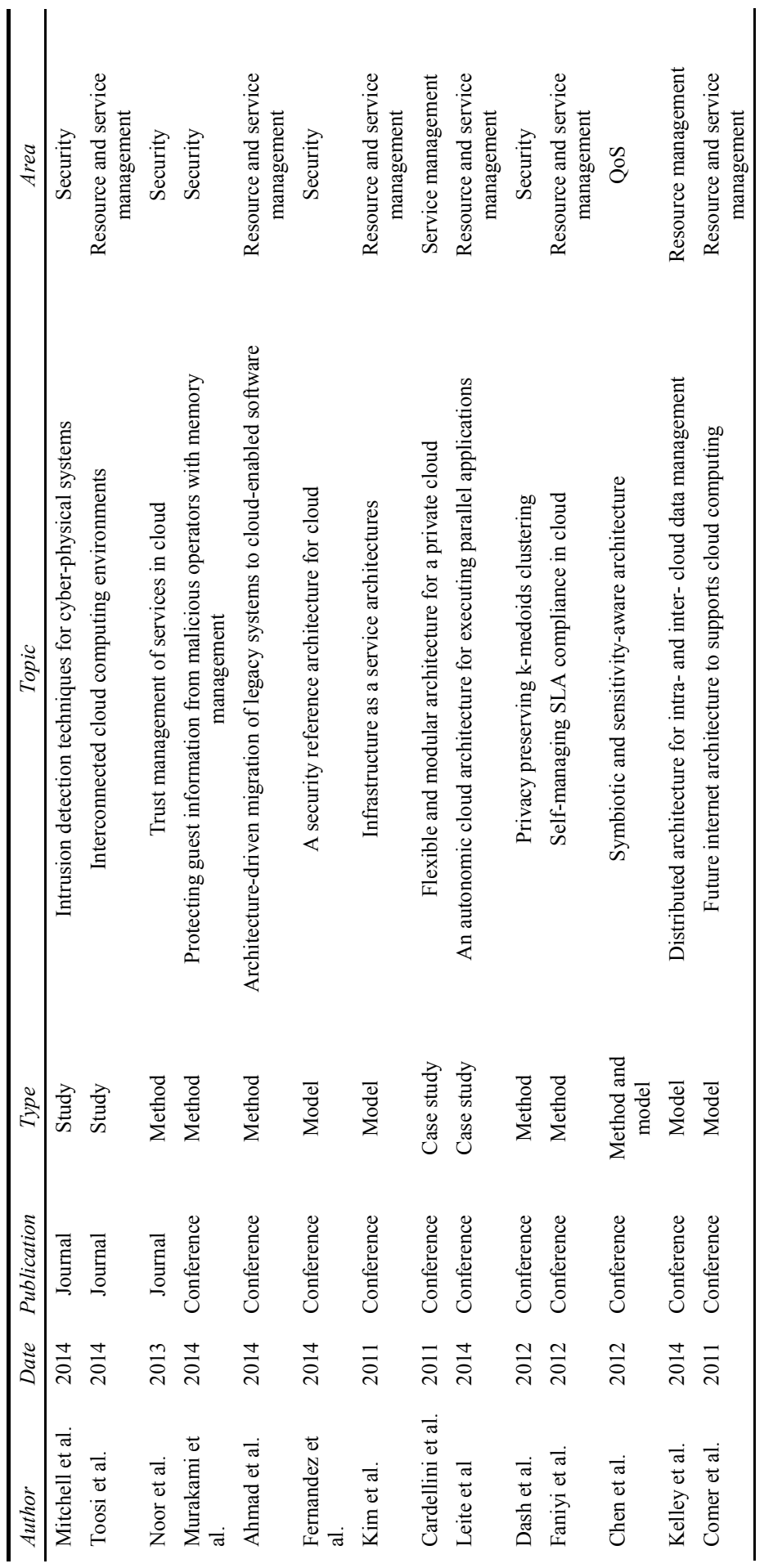


Table 5 Included results from ACM (continued)

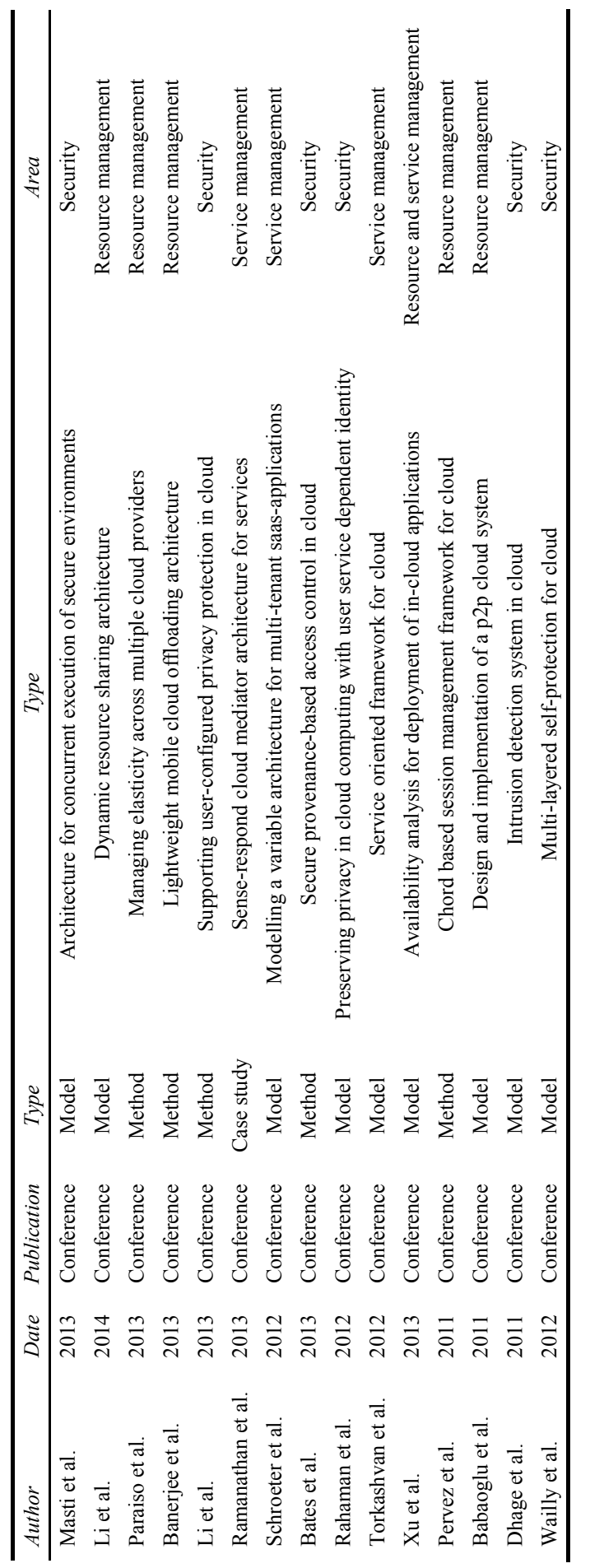


Table 6 Included results from science direct

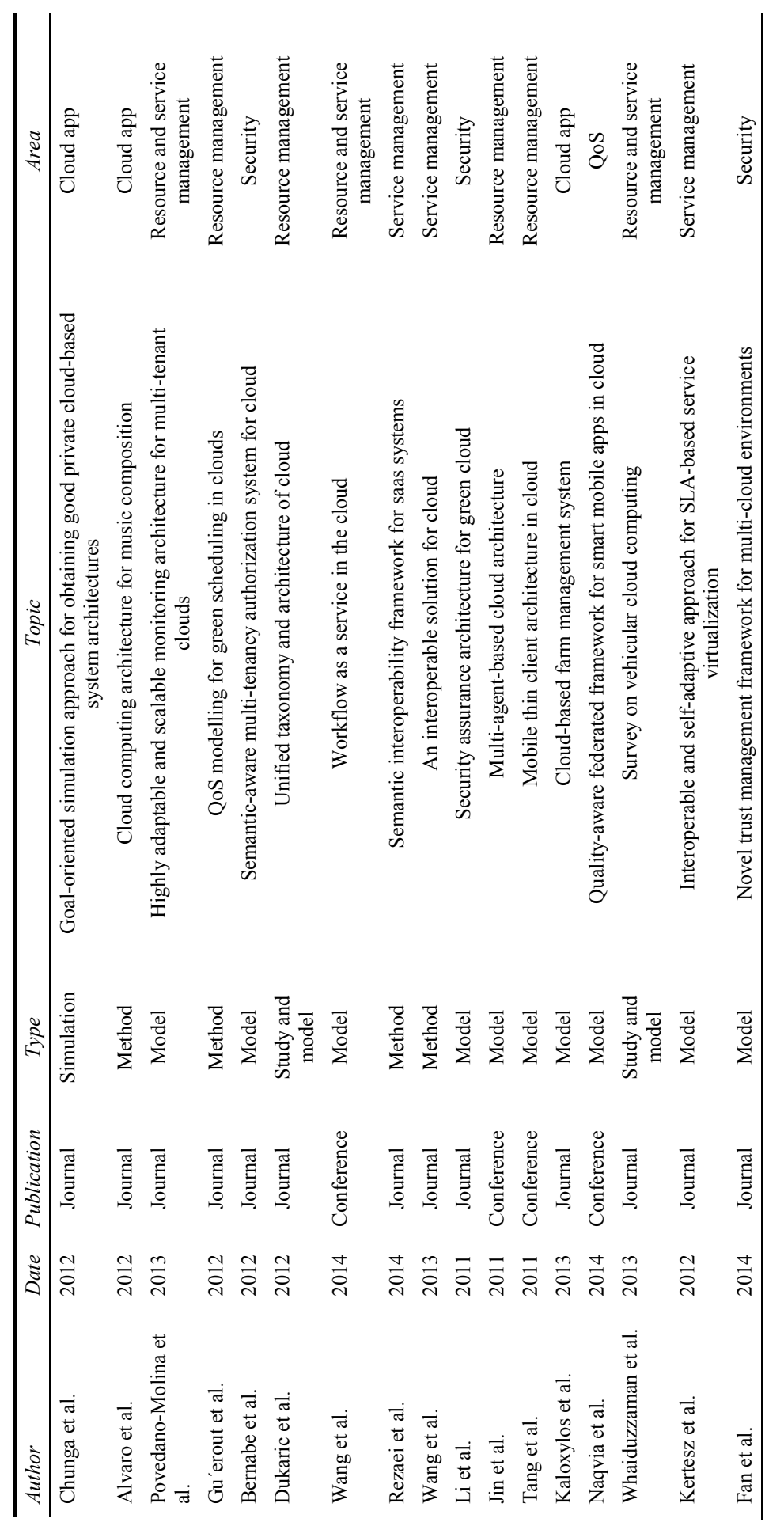


Table 6 Included results from science direct (continued)

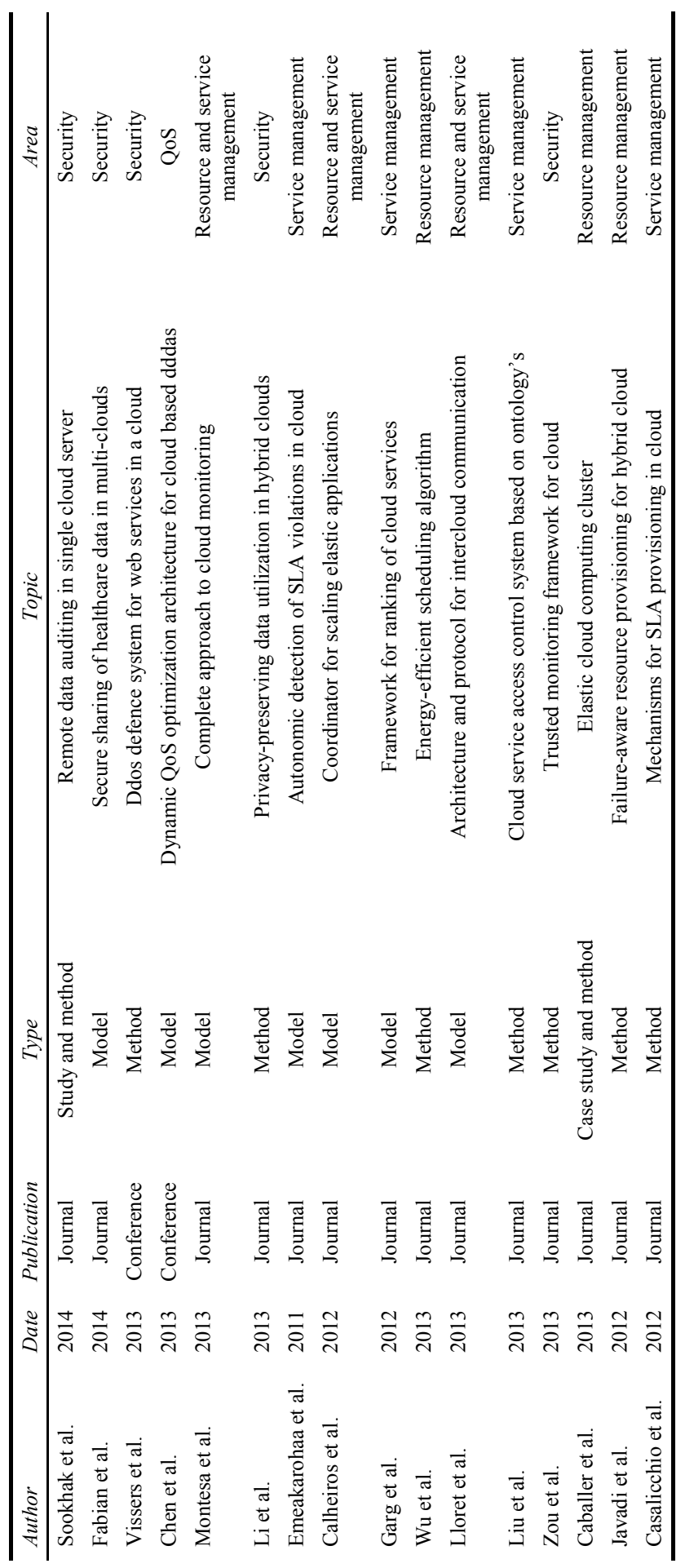


Table 7 Included results from Springer

\begin{tabular}{|c|c|c|c|c|c|}
\hline Author & Date & Publication & Type & Topic & Area \\
\hline Khan et al. & 2012 & Journal & Model & $\begin{array}{l}\text { Integrating intelligence in } \\
\text { urban management }\end{array}$ & Cloud app \\
\hline Wang et al. & 2012 & Journal & Study & $\begin{array}{l}\text { Enterprise cloud service } \\
\text { architectures }\end{array}$ & $\begin{array}{c}\text { Service } \\
\text { management }\end{array}$ \\
\hline $\begin{array}{l}\text { Colombo-Mendoza } \\
\text { et al. }\end{array}$ & 2014 & Journal & $\begin{array}{c}\text { Model } \\
\text { and app }\end{array}$ & $\begin{array}{l}\text { Paas for cloud services- } \\
\text { based mobile applications }\end{array}$ & $\begin{array}{l}\text { Model and cloud } \\
\text { app }\end{array}$ \\
\hline Muñoz et al. & 2013 & Journal & Model & $\begin{array}{l}\text { Constructing resilient } \\
\text { services on federated } \\
\text { hybrid clouds }\end{array}$ & $\begin{array}{c}\text { Service } \\
\text { management }\end{array}$ \\
\hline Qi et al. & 2014 & Journal & Model & $\begin{array}{l}\text { Sierpinski triangle-based } \\
\text { data centre architecture }\end{array}$ & $\begin{array}{c}\text { Resource } \\
\text { management }\end{array}$ \\
\hline Chapman et al. & 2011 & Journal & Model & $\begin{array}{l}\text { Architecture for on- } \\
\text { demand cloud } \\
\text { provisioning }\end{array}$ & $\begin{array}{l}\text { Service and } \\
\text { resource } \\
\text { management }\end{array}$ \\
\hline Hussain et al. & 2014 & Journal & Model & $\begin{array}{c}\text { Software quality in the } \\
\text { clouds }\end{array}$ & Qos and security \\
\hline Hu et al. & 2011 & Journal & Model & $\begin{array}{l}\text { Green private cloud } \\
\text { architecture }\end{array}$ & Qos \\
\hline Chen et al. & 2011 & Journal & $\begin{array}{l}\text { Model } \\
\text { and } \\
\text { method }\end{array}$ & $\begin{array}{l}\text { Integrated management of } \\
\text { diverse cloud resources }\end{array}$ & $\begin{array}{c}\text { Resource } \\
\text { management }\end{array}$ \\
\hline Vilaplana et al. & 2014 & Journal & Method & $\begin{array}{l}\text { Queuing theory model for } \\
\text { cloud }\end{array}$ & Qos \\
\hline Beach et al. & 2013 & Journal & Method & $\begin{array}{l}\text { Cloud architecture for } \\
\text { engineering \& } \\
\text { construction sector: }\end{array}$ & Cloud app \\
\hline Ros et al. & 2014 & Journal & Method & $\begin{array}{l}\text { Cloud architecture for } \\
\text { web applications with } \\
\text { load forecasting } \\
\text { mechanism }\end{array}$ & $\begin{array}{c}\text { Resource } \\
\text { management }\end{array}$ \\
\hline Perez-Sorrosal et al. & 2011 & Journal & Method & $\begin{array}{l}\text { Consistent and scalable } \\
\text { caching in multi-tier } \\
\text { architectures }\end{array}$ & $\begin{array}{c}\text { Resource } \\
\text { management }\end{array}$ \\
\hline Joshi et al. & 2014 & Journal & Method & $\begin{array}{l}\text { Fault tolerance } \\
\text { mechanisms for virtual } \\
\text { data centre architectures }\end{array}$ & $\begin{array}{c}\text { Resource } \\
\text { management }\end{array}$ \\
\hline
\end{tabular}

RQ4 What is the different application domains/case studies implemented?

We classified publications as case studies when they were explicitly specified in the abstract. The study (Caballer et al., 2013) assessed the effectiveness of an elastic virtual cluster on a cloud infrastructure, tries to analyse the usage of EC3 solution and to execute an HTC-based scientific application. A similar study (Cardellini and Iannucci, 2012) implements Linux terminal server project (LTSP) which is a free and open source terminal server for Linux that allows many users to simultaneously use the same computer. Another study (Leite et al., 2014; Zhou et al., 2015) executes without auto scaling, by simulating user preferences, where an instance is selected either upon their 
knowledge or the amount of computational resources offered by an instance, it also evaluates the architecture that will scale for a cloud unaware application. Study by Ramanathan et al. (2011) illustrates an adaptive complex environment and it accommodates service progression from simple to the complex, achieving the overall mayoral goals to make a city the finest place to live, work, and raise a family. The case studies imply that there is still relatively little research in having cloud applications. The application domains are listed in Table 9.

Figure 5 Weighted research topic cloud

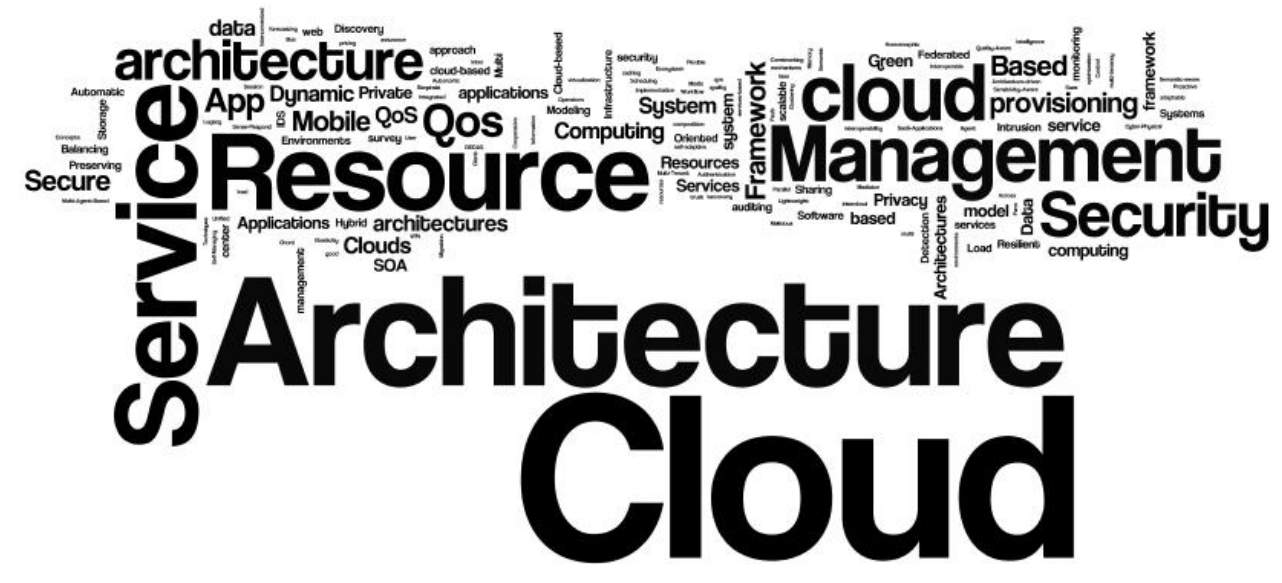

Figure 6 Taxonomy of research topics (see online version for colours)

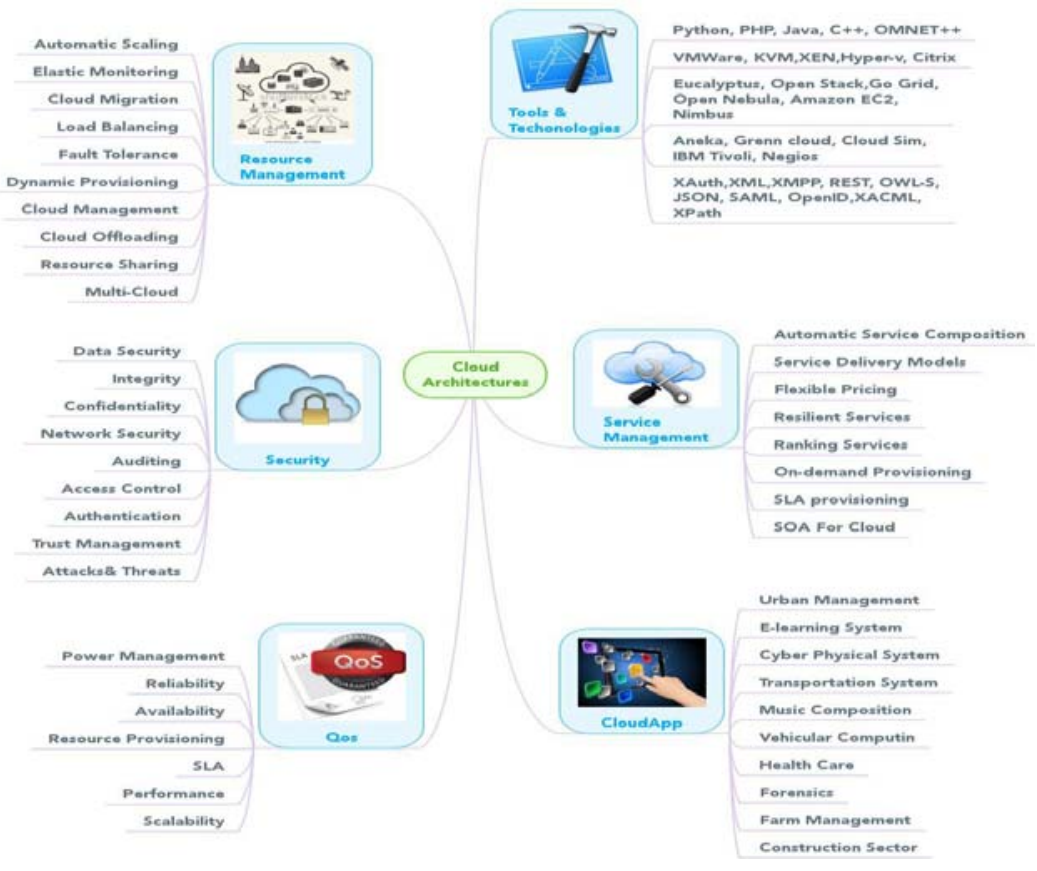


Table 8 Tools and technologies

\begin{tabular}{|c|c|}
\hline Category & Tools, standards and technologies \\
\hline $\begin{array}{l}\text { Resource } \\
\text { management }\end{array}$ & $\begin{array}{l}\text { Python, Oracle Virtual Box, OAuth, XML, MySQL, health insurance } \\
\text { portability and accountability act (HIPAA), Amazon EC2, Microsoft Azure, } \\
\text { Xen, PHP, Google App Engine (GAE), Nagios, Gangila, Command } \\
\text { Description Language (CDL), Groovy, Smart Frog, GoGrid, Open Stack, } \\
\text { Aneka, Open Nebula, REST, Resource Description Framework (RDF), } \\
\text { Scrappy, XPath, DOM, WADL, Template Design Language (TDL), Service } \\
\text { Provider Markup Language (SPML), XMPP, XACML, SAML, } \\
\text { VmwareVcloud, Cloud Sigma, JCloud, Open Science Data Cloud (OSDC), } \\
\text { IBM Blue Cloud, Open Virtualization Format (OVF), Essential Meta -Object } \\
\text { Facility (EMOF), Java, C++, HUTN (Meta Language), Business Process } \\
\text { Execution Language (BPEL), VMWare, Xen. }\end{array}$ \\
\hline \multirow[t]{3}{*}{$\begin{array}{l}\text { Service } \\
\text { management }\end{array}$} & $\begin{array}{l}\text { Java, NASC Development Kit, OWL-S (Semantic Web Service Composition } \\
\text { Framework), Web Service Modeling Ontology (WSMO), Web Service } \\
\text { Modeling Language WSML, }\end{array}$ \\
\hline & $\begin{array}{l}\text { IBM Tivoli, HP Network Management Center, Nagios, Splunk, Jasper Report, } \\
\text { Distributed Management Task Force (DMTF), Web based Enterprise } \\
\text { Management (WBEM), Cloud Infrastructure Management Interface (CIMI), } \\
\text { SPML, Nimbus, JSON, PHP, WS -BPEL (Business Process Execution } \\
\text { Language), Java Messaging Service (JMS) API, }\end{array}$ \\
\hline & $\begin{array}{l}\text { Sniffer pro 4.70.04, Resource and Application Description Language (RADL), } \\
\text { Kepler, Django. }\end{array}$ \\
\hline $\begin{array}{l}\text { Quality of } \\
\text { service }\end{array}$ & $\begin{array}{l}\text { WS-Agreement Specification, OMNET++, OpenVZ, Eucalyptus, Universal } \\
\text { Description, Discovery and Integration (UDDI), IBM Tivoli Manager, JOnAS } \\
\text { Java EE Application Server, CloudSim, Sage 5.3 Mathematical Software, } \\
\text { Apache Jmeter. }\end{array}$ \\
\hline Security & $\begin{array}{l}\text { Security Assertion Markup Language (SAML), OpenID, Ontology Web } \\
\text { Language (OWL), Sematic Web Rule Language (SWRL), FaCT++, Pellet, } \\
\text { Payment Card Industry Data Security Standard (PCI DSS), FISMA (US Fedral } \\
\text { Information Security Management Act), FedRAMP (Fedral Risk and } \\
\text { Authorization Management Program), XACML, Host Based Security Tools } \\
\text { (HBST), Intrusion Detection Message Exchange Format (IDMEF), Cloud } \\
\text { Visor. }\end{array}$ \\
\hline $\begin{array}{l}\text { Cloud } \\
\text { applications }\end{array}$ & $\begin{array}{l}\text { SOAP,WSDL,KVM,XML-RPC, JSON, PHP, AJAX, Cloud BIM, Cloudsim, } \\
\text { JSON, LISP, Python, SOA, }\end{array}$ \\
\hline
\end{tabular}

RQ5 What are the different cloud setups that have been adopted?

Cloud setup is a platform for experimentation of large development projects. We find various cloud setups that were used in our studies listed in Table 10. This setup uses different tools and technologies (see Table 8) for different purposes, but this body of knowledge provides to setup our own cloud environment with respective to our experiments. 
Table 9 Application domains

\begin{tabular}{lc}
\hline Application domains & Reference \\
\hline Transportation system & Chung et al. (2012) \\
Music composition & Alvaro and Barros (2012) \\
Farm management & Kaloxylos et al. (2013) \\
Healthcare & Fabian et al. (2014), Fortino et al. (2014) \\
Urban management & Khan et al. (2012) \\
E-learning system & Ros et al. (2014) \\
Construction sector & Beach et al. (2013) \\
Vehicular computing & Luo et al. (2012) \\
Video streaming & Thorpe et al. (2013) \\
Forensic & Ramanathan et al. (2011) \\
E-government & Yao et al. (2013) \\
E-commerce & Dukaric and Juric (2012) \\
Cyber physical system & Colombo-Mendoza et al. (2014) \\
Enterprise cloud service & Heyong Wang et al. (2012) \\
Mobile application & (1998) \\
\hline
\end{tabular}

\section{Discussion}

This section provides a discussion of the results and limitations for this study.

\subsection{Conclusions on the state of the art}

After synthesising data collected through this SLR, we observed number of research trends in resource management, service management, quality of service, security, CloudApp and few research challenges were not addressed properly. The maturity of cloud architectures is still in its early stages.

However, we can find a clear growth in maturity and researchers need to focus on a vertical approach. More case studies will improve the confidence of researchers and practitioners regarding the benefits of cloud architectures.

\subsection{Conclusions for a body of knowledge}

After analysing the results of SLR, the body of knowledge has areas that represent cloud architectures which deals with resource management, service management, quality of service, security, cloud-app and various tools and technologies those are used in the study. This is illustrated in Figure 7. 
Table 10 Cloud setups

\begin{tabular}{|c|c|}
\hline Study reference & Cloud setup \\
\hline $\begin{array}{l}\text { Xu and Li and } \\
\text { (2013) }\end{array}$ & $\begin{array}{l}\text { - } 20 \text { Dual-Core intelxeon 3.0 GHZ connected through Gigabit Ethernet } \\
\text { - Each machine has 2GB Memory } \\
\text { - Each VM have 1.5 GHZ CPU and 256 MB RAM. } \\
\text { - All machines run Ubuntu 8.04.4 LTS with Linux 2.6.24-28 Server } \\
\text { - A cluster of Dual intelxeon 2.4 GHZ servers are used to generate } \\
\text { workload } \\
\text { - Anchor machine with Ubuntu } 8.10 \text { server, Apache 2.2.9, PHP 5.2.6 } \\
\text { and Mysql 5.0.6.7 }\end{array}$ \\
\hline $\begin{array}{l}\text { Zhen Xiao et al. } \\
\text { (2014) }\end{array}$ & $\begin{array}{l}\text { Load Shifting } \\
\text { - Three servers and three applications } \\
\text { - Each server with Intel E5420 CPU, 8 GB RAM and run on Xen 3.3.1 } \\
\text { Auto Scaling } \\
\text { - } 30 \text { Dell power edge servers with Intel E5620 CPU, } 24 \text { GB RAM and } 9 \\
\text { applications } \\
\text { - Server runs on xen-4.0 and Linux 2.6.18 }\end{array}$ \\
\hline Misra et al. (2014) & $\begin{array}{l}\text { - } 3 \text { vm's in Bangalore with nimbus } 2.9 \text { infrastructure. } \\
\text { Hardware Specification } \\
\text { Intel xeon 3.16GHZ, 1GB RAM, 100GB HD. } \\
\text { Software Specification } \\
\text { RHEL 5.1, Intel MK Libraries and Netcdf libraries. } \\
\text { - } 2 \text { vm's in Hyderabad with nimbus } 2.9 \text { infrastructure. } \\
\text { Hardware Specification } \\
\text { Intel xeon 3.16GHZ, 1GB RAM, 120GB HD. } \\
\text { Software Specification } \\
\text { Scientific Linux } \\
\text { - } 2 \text { vm's in Chennai with nimbus } 2.9 \text { infrastructure. } \\
\text { Hardware Specification } \\
\text { Intel xeon 3.16GHZ, 1GB RAM, 80GB HD. } \\
\text { Software specification } \\
\text { Centos, Intel MK Libraries }\end{array}$ \\
\hline
\end{tabular}


Table 10 Cloud setups (continued)

\begin{tabular}{|c|c|}
\hline Study reference & Cloud setup \\
\hline $\begin{array}{l}\text { Varadharajan and } \\
\text { Tupakula (2014) }\end{array}$ & $\begin{array}{l}\text { - Xen hypervisor } \\
\text { - Vm Dom } 0 \text { is used for hosting and management of virtual machine } \\
\text { - } 1 \mathrm{Vm} \text { based on Linux and another windows Vm running on xen } \\
\text { - SPECJvm benchmark were installed on DOM } 0 \text { of xen with following } \\
\text { specifications, Intel i7 } 2.2 \mathrm{GHZ}, 6 \mathrm{M} \text { cache with } 8 \text { GB RAM, Xen } 3.12 \\
\text { VMM, centos } 5.1 \\
\text { - Vm running with windows xp sp2 and linuxos with } 512 \text { MB RAM }\end{array}$ \\
\hline $\begin{array}{l}\text { Munoz et al. } \\
\text { (2013) }\end{array}$ & $\begin{array}{l}\text { - The testing setup has } 250 \text { vm's in which } 10 \text { vm's for USC, } 90 \text { vm's for } \\
\text { PIC and } 150 \text { vm's for CC.IN 2P3 } \\
\text { - PIC hosts are BL460C Blade with } 8 \text { cores intelxeon L5420, } 2.50 \mathrm{GHZ} \\
\text { and } 16 \text { GB RAM } \\
\text { - CC.IN2P3 hosts are Dell Power Edge C6100 systems with } 24 \text { cores } \\
\text { intelxeon X5675 } 3.07 \text { GHZ and } 96 \text { GB RAM } \\
\text { - USC hosts are AMD } 6400 \text { MT with } 16 \text { cores AMD Opteron } 6128 \\
\text { magany } 2.0 \text { GHZ and } 16 \text { GB RAM }\end{array}$ \\
\hline $\begin{array}{l}\text { Perez-Sorrosal } \\
\text { et al. (2011) }\end{array}$ & $\begin{array}{l}\text { - Cluster of } 10 \text { machines connected through 100mbps switch, each node } \\
\text { have } 2 \text { AMD Athlon 2GHZ CPU's, 1GB RAM, two } 320 \text { GB HD and } \\
\text { runFedro Linux } \\
\text { - JEE application server } \\
\text { - JonAS v.4.7.1 application server, postgresql v.8.2.1 and SPECjApp } \\
\text { server } 2004 \\
\text { - JGroup replication protocol, Apache HTTP Server }\end{array}$ \\
\hline $\begin{array}{l}\text { Kaloxylos et al. } \\
\text { (2013) }\end{array}$ & 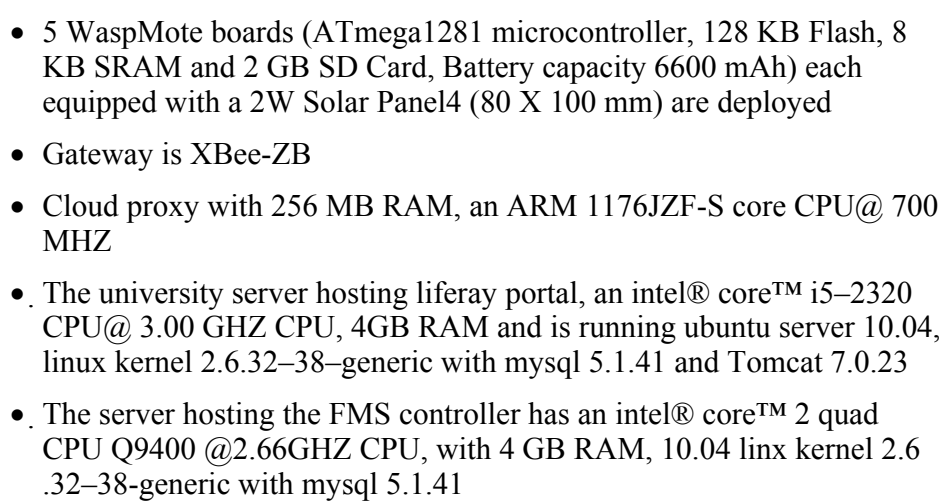 \\
\hline
\end{tabular}


Table 10 Cloud setups (continued)

\begin{tabular}{|c|c|}
\hline Study reference & Cloud setup \\
\hline Montes et al. (2013) & $\begin{array}{l}\text { - Experiments are carried out on Grid } 5000 \\
\text { - } 45 \text { nodes from suno cluster on the Sophia site } \\
\text { Physical Resources } \\
\text { - } 45 \text { cluster nodes outfitted with debian GNU/linuxlenny with kernel } \\
2.6 .32 \text {, x86-64 intelxeon E55520 2.26GHZ CPU's, 32 GB of } \\
\text { RAM and 2GB Ethernet (Broadcom Netxtremell BCM5716) } \\
\text { Cloud Iaas } \\
\text { - OpenNebula } \\
\text { Virtual Resources } \\
\text { - } 80 \text { vm's outfitted with debian GNU/linuxlenny with kernel 2.6.32, } \\
\text { a single virtual x86-64 intelxeon E55520 2.26GHZ CPUs, 1 GB of } \\
\text { RAM } \\
\text { - } 80 \text { vm's are deployed at client side }\end{array}$ \\
\hline Calheiros et al. (2012) & $\begin{array}{l}\text { - One cloud exchange and two cloud coordinators are deployed in } \\
\text { GlassFish V3 application server } \\
\text { - Cloud coordinators have comprised of three xeonquard core } 2.00 \\
\text { GHZ processors with 8GB of RAM and two } 160 \text { GB HD (mirrored } \\
\text { RAID1) } \\
\text { - Management of virtual resources are taken care by Eucalyptus } \\
1.6 .2 \text { that runs on one server, two servers are hosting vm's } \\
\text { - Second cloud coordinator mediates access to Amazon EC2 cluster } \\
\text { - Three types of instances } \\
\text { - Small ( } 1 \text { core, } 1.7 \mathrm{~GB} \text { RAM, } 1 \mathrm{EC} 2 \text { computing unit and costing } \$ \\
\text { - Large (2 cores, } 7.5 \mathrm{~GB} \text { RAM, } 2 \mathrm{EC} 2 \text { computing unit and costing \$ } \\
\text { - Lanstance per hour) } \\
\text { - Extra large (4 cores, } 15 \mathrm{~GB} \text { RAM, } 2 \mathrm{EC} 2 \text { computing unit and } \\
\text { costing \$ } 0.76 \text { per instance per hour) }\end{array}$ \\
\hline Lloret et al. (2013) & $\begin{array}{l}\text { - Cloud exchange and cloud coordinator B run in an intel core2 } \\
\text { 6600(Dual core, 2.4GHZ) with 2GB RAM, } 70 \text { GB HD } \\
\text { - Cloud coordinator a run in an intel core2 Duo E8400 (Dual core, } \\
\text { 3GHZ) with 3GB RAM, } 140 \text { GB HD } \\
\text { - Cloud topology composed by } 24 \text { computers with Intel Celeron } 2 \\
\text { GHZ, } 256 \text { GB of RAM and 100BaseT links are used } \\
\text { - The operating system is windows XP } \\
\text { - To capture data Sniffer pro } 4.70 .04 \text { is used }\end{array}$ \\
\hline
\end{tabular}


Figure 7 Areas of the body of knowledge for cloud architectures in cloud (see online version for colours)

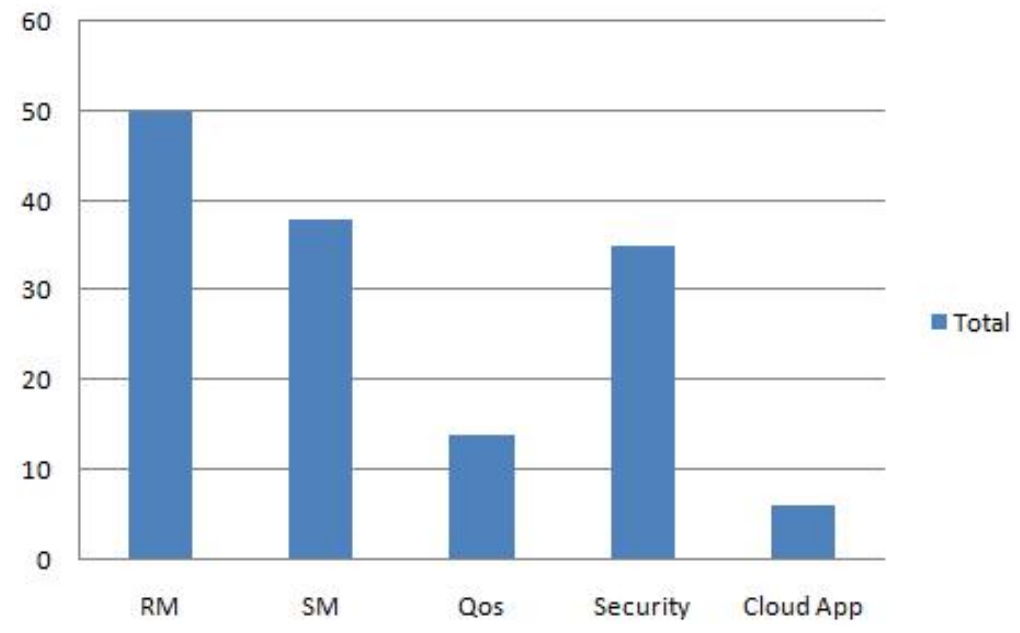

\subsection{Threats to validity}

This SLR provides a study of software architectures for cloud computing. Though the results of reviews are reliable, they have potential threats to validity. The main threats of this review are the bias in our selection of studies to be included, data extraction and synthesis. In order to mitigate potential threats to validity, we define a research protocol, which contains research questions, inclusion/exclusion criteria, research strategy and followed the guidelines of a systematic review (Kitchenham and Charters, 2007). In our search strategies, the main idea was to regain as much as possible of the available literature to avoid any bias. Cloud architectures relate to different computer science communities in order to cover all and avoid bias. We searched for common terms and combined them in our search string, which decreases bias and increases search work.

The research protocol was developed by the first author and was reviewed by the second author, to ensure the review selection process and the search string was derived from research questions. To ensure correctness in data extraction, we defined a Meta document which contains consistent and relevant data with respective to search string, inclusion/exclusion criteria and research questions. In order to mitigate reliability threat several researchers are involved in reviewing the included papers to achieve high validity of the study.

\section{Conclusions}

The objective of this study was to consolidate existing research on cloud architectures and associated topics that allow for building up a body of knowledge. We considered 121 out of 400 reviewed publications significant with respect to research protocols, research question and categorised them according to the research area. On that basis, we provided taxonomy for representing research areas, application domain, tools and technologies. We identified unexplored areas by synthesising collected data, making those available for 
future research. We observed vast interests towards resource management, service management and security areas. We also observed a lack of tools and also lack of evidence for architectural adaption to develop common and secure architecture. The field is still in its early stages and in order to mature, cloud computing and software engineering researchers should come together by proposing a common research agenda.

\section{References}

Ali, M., Khan, S.U. and Vasilakos, A.V. (2015) 'Security in cloud computing: opportunities and challenges', Information Science, Vol. 305, No. 5, pp.357-383.

Alvaro, J.L. and Barros, B. (2012) 'A new cloud computing architecture for music composition', Journal of Network and Computer Applications, Vol. 36, No. 1, pp.429-443.

Bass, C. and Kazman (2012) Software Architecture in Practice, 3rd ed., Addison-Wesley Professional Publisher, Boston, USA.

Beach, T.H., Rana, O.F., Rezguil, Y. and Parashar, M. (2013) 'Cloud computing for the architecture, engineering \& construction sector: requirements, prototype \& experience', Journal of Cloud Computing: Advances, Systems and Applications, Vol. 2, No. 8, pp.1-16.

Blank, R.M. (2011) The NIST Definition of Cloud Computing, pp.800-145, National Institute of Standards and Technology, USA.

Buyya, R., Yeo, C.S., Venugopal, S. Broberg, J. and Brandic, I. (2009) 'Cloud computing and emerging IT platforms: vision, hype, and reality for delivering computing as the 5th utility', Future Generation Computer Systems, Vol. 25, No. 6, pp.599-616.

Caballer, M., de Alfonso, C., Alvarruiz, F. and Molto, G. (2013) 'EC3: elastic cloud computing cluster', Journal of Computer and System Sciences, Vol. 79, No. 8, pp.1341-1351.

Calheiros, R.N., Toosi, A.N., Vecchiola, C. and Buyya, R. (2012) 'A coordinator for scaling elastic applications across multiple clouds', Future Generation Computer Systems, Vol. 28, No. 4, pp.1350-1362.

Cardellini, V. and Iannucci, S. (2012) 'Designing a flexible and modular architecture for a private cloud: a case study', Proceedings of the 6th International Workshop on Virtualization Technologies in Distributed Computing Date, pp.37-44.

Chauhan, M.A. (2014) A Systematic Mapping Study of Software Architectures for Cloud Based Systems, Unpublished PhD thesis, IT university of Copenhagen, Denmark.

Chung, L., Hill, T., Legunsen, O., Sun, Z., Dsouza, A. and Supakkul, S. (2012) 'A goal-oriented simulation approach for obtaining good private cloud-based system architectures', The Journal of Systems and Software, Vol. 86, No. 9, pp.2242-2262.

Colombo-Mendoza, L.W., Alor-Hernández, G., Rodríguez-gonzález, A. and Valencia-garcía, R. (2014) 'MobiCloUP!: a PaaS for cloud services-based mobile applications', Autom. Softw. Eng., Vol. 21, No. 3, pp.391-437.

Duan, Q., Yan, Y., Vasilakos, A.V. (2012) 'A survey on service-oriented network virtualization toward convergence of networking and cloud computing', IEEE Transactions on Network and Service Management, Vol. 9, No. 4, pp.373-392.

Dukaric, R. and Juric, M.B. (2012) 'Towards a unified taxonomy and architecture of cloud frameworks', Future Generation Computer Systems, Vol. 29, No. 5, pp.1196-1210.

Fabian, B., Ermakova, T. and Junghanns, P. (2014) 'Collaborative and secure sharing of healthcare data in multi-clouds', Information Systems, Vol. 48, No. 5, pp.132-150.

Fortino, G., Di Fatta, G., Pathan, M. and Vasilakos, A.V. (2014) 'Cloud-assisted body area networks: state-of-the-art and future challenges', Wireless Networks, Vol. 20, No. 7, pp.1925-1938. 
Kaloxylos, A., Groumas, A.., Sarri, V., Katsikas, L., Magdalinos, P., Antoniou, E., Politopoulou, Z., Wolfert, S., Brewster, C., Eigenmann, R. and Terol, C.M. (2013) 'A cloud-based farm management system: architecture and implementation', Computers and Electronics in Agriculture, Vol. 100, No. 9, pp.168-179.

Khan, Z., Ludlow, D., McClatchey, R. and Anjum, A. (2012) 'An architecture for integrated intelligence in urban management using cloud computing', Journal of Cloud Computing: Advances, Systems and Applications, Vol. 1, No. 1, pp.1-14.

Kitchenham, B. and Charters, S. (2007) Guidelines for Performing Systematic Literature Reviews in Software Engineering, Software Engineering Group, Keele University, UK.

Leite, A.F., Tadonki, C. and Eisenbeis, C. (2014) 'Excalibur: an autonomic cloud architecture for executing parallel applications', Proceedings of the Fourth International Workshop on Cloud Data and Platforms, pp.122-128.

Liu, F. et al. (2011) 'NIST cloud computing reference architecture', National Institute of Standards and Technology, U.S Department of Commerce, Special Publication, pp.500-292.

Lloret, J., Garcia, M., Tomas, J. and Joel, J.P.C. and Rodrigues (2013) 'Architecture and protocol for intercloud communication', Information Sciences, Vol. 258, No. 8, pp.434-451.

Luo, H., Egbert, A. and Stahlhut, T. (2012) 'QoS architecture for cloud-based media computing', Proceedings of the IEEE 3rd International Conference on Software Engineering and Service Science, pp.769-772.

Misra, S., Krishna, V.P., Kalaiselvan, K., Saritha, V. and Mohammad Obaidat, S. (2014) 'Learning automata-based QoS framework for cloud IaaS', IEEE Transactions on Network and Service Management, Vol. 11, No. 1, pp.15-24.

Mitchell, R. and Chen, I-R. (2014) 'A survey of intrusion detection techniques for cyber-physical systems', ACM Computing Surveys, Vol. 46, No. 4, pp.55.1-55.29.

Montes, J., Sánchez, A., Memishi, B., Pérez, M.S. and Antoniu, G. (2013) 'GMonE: a complete approach to cloud monitoring', Future Generation Computer Systems, Vol. 29, No. 8, pp.2026-2040.

Muñoz, V.M., Ramo, A.C., Albor, V.F., Graciani, R. and Arévalo, G.M. (2013) 'Rafhyc: an architecture for constructing resilient services on federated hybrid clouds', J. Grid Computing, Vol. 11, No. 4, pp.753-770.

Perez-Sorrosal, F., Patiño-Martinez, M., Jimenez-Peri, R. and Kemme, B. (2011) 'Elastic SI-Cache: consistent and scalable caching in multi-tier architectures', The VLDB Journal, Vol. 20, No. 6, pp.841-865.

Ramanathan, J., Ramnath, R., Singh, N., Xu, Z. and Xu, Y. (2011) 'Sense-respond cloud mediator architecture for services evolution', Proceedings of the 2011 ACM Symposium on Applied Computing, pp.162-169.

Ros, S., Caminero, A.C., Hernández, R., Robles-Gómez, A. and Tobarra, L. (2014) 'Cloud-based architecture for web applications with load forecasting mechanism: a use case on the e-learning services of a distant university', J. Supercomput., Vol. 68, No. 3, pp.1556-1578.

Stifani, R., Pappe, S., Breiter, G. and Behrendt, M. (2012) 'IBM cloud computing reference architecture', IBM Academy of Technology, Academy TechNotes, ATN, Vol. 3.

Thorpe, S., Grandison, T., Campbell, A., Williams, J., Burrell, K. and Ray, I. (2013) 'Towards a forensic-based service oriented architecture framework for auditing of cloud logs', Proceedings of the IEEE Ninth World Congress on Services.

Varadharajan, V. and Tupakula, U. (2014) 'Security as a service model for cloud environment', IEEE Transactions on Network and Service Management, Vol. 11, No. 1, pp.60-75.

Vasilakos, A., Ricudis, C., Anagnostakis, K., Pedryca, W. and Pitsillides, A (1998) 'Evolutionaryfuzzy prediction for strategic QoS routing in broadband networks', IEEE International Conference on Fuzzy Systems Proceedings, Vol. 2, No. 1, pp.1488-1493.

Vasilakos, A.V. (2008) 'Ambiet intelligence', Information Sciences, Vol. 178, No. 3, pp.585-587.

Wang, H., He, W. and Wang, F-W. (2012) 'Enterprise cloud service architectures', Inf. Technol. Manag, Vol. 13, No. 4, pp.445-454. 
Whaiduzzaman, Md., Sookhak, M., Gain, A. and Buyya, R. (2013) 'A survey on vehicular cloud computing', Journal of Network and Computer Applications, Vol. 40, No. 5, pp.325-344.

Xiao, Z., Chen, Q. and Luo, H. (2014) 'Automatic scaling of internet applications for cloud computing services', IEEE Transactions on Computers, Vol. 63, No. 5, pp.1111-1123.

Xu, F., Liu, F., Jin, H. and Vasilakos, A.V. (2014) 'Managing performance overhead of virtual machines in cloud computing: a survey, state of the art, and future directions,' Proceedings of the IEEE, Vol. 102, No. 1, pp.11-31.

$\mathrm{Xu}, \mathrm{H}$. and Li, B. (2013) 'Anchor: a versatile and efficient framework for resource management in the cloud', IEEE Transactions on Parallel and Distributed Systems, Vol. 24, No. 6, pp.1066-1076.

Yao, J.T., Vasilakos, A.V. and Pedrycz, W. (2013) 'Granular computing: perspectives and challenges', Neurocomputing, Vol. 7, No. 3, pp.275-297.

Zhou, C., Quach, D-C., Xiong, N., Huang, S., Zhang, Q., Yin, Q. and Vasilakos, A.V. (2015) 'An improved direct adaptive fuzzy controller of an uncertain PMSM for web-based e-service systems', IEEE T. Fuzzy Systems, Vol. 23, No. 1, pp.58-71. 